\title{
ORDER-TOPOLOGICAL LATTICES
}

\author{
by MARCEL ERNÉ
}

(Received 31 October, 1978)

1. Introduction and basic concepts. The observation that convergence of real sequences may be defined in terms of limits inferior and limits superior as by means of neighbourhoods in the Euclidean topology leads to the question: for which lattices does order convergence coincide with convergence in the order topology? This problem has been attacked by D. C. Kent [10], A. Gingras [7] and others. We hope to present a satisfactory solution in this paper. Although there are known several characterizations of lattices. with topological order convergence (cf. Propositions 1,2), an evaluation of these criteria already requires some knowledge of the order topology of the given lattice. In the present paper, we establish a purely lattice-theoretical description of those lattices for which order convergence is not only topological, but moreover, the lattice operations are continuous. Henceforth, such lattices will be referred to as order-topological lattices. All convergence statements will be formulated in terms of filters rather than nets. For an introduction to convergence functions, the reader may consult D. C. Kents's paper [9].

Let $L$ be any lattice, partially ordered by an order relation $\leq$. For a subset $Y$ of $L$, let $Y^{\downarrow}$ and $Y^{\uparrow}$ denote the set of all lower and upper bounds for $Y$, respectively. If $x$ is the join (i.e. the least upper bound) of $Y$ then we indicate this by the symbol $x=\vee Y$. Similarly, we write $x=\bigwedge Y$ if $x$ is the meet (i.e. the greatest lower bound) of $Y$.

Furthermore, $x=y \vee z$ means $x=\bigvee\{y, z\}$, and $x=y \wedge z$ means $x=\wedge\{y, z\}$. For the sake of convenience, we set

$$
\begin{aligned}
& x \wedge Y=\{x \wedge y: y \in Y\} \\
& Y \wedge Z=\{y \wedge z: y \in Y, z \in Z\}
\end{aligned} \quad(x \in L ; Y, Z \subseteq L)
$$

Finally, for any filter $\mathfrak{\mho}$ on $L$, we write

$$
\mathfrak{F}^{\downarrow}=\bigcup\left\{F^{\downarrow}: F \in \mathfrak{F}\right\}, \quad \mathfrak{F}^{\uparrow}=\bigcup\left\{F^{\uparrow}: F \in \mathfrak{F}\right\} .
$$

$\Im$ order converges to a point $x \in L$ (written $\mathfrak{\mho} \longrightarrow x$ ) if

$$
x=\bigvee \mathfrak{F}^{\downarrow}=\bigwedge \mathfrak{F}^{\uparrow}
$$

For all $x \in L$, the intersection

$$
\mathfrak{B}(x)=\bigcap\{\mathfrak{F}: \mathfrak{F} \longrightarrow x\}
$$

is again a filter on $L$, but $\mathfrak{B}(x)$ need not order converge to $x$ :

Example 1. The set

$$
L=\{(x, 0): 0 \leqslant x<1\} \cup\{(0, y): 0 \leqslant y<1\} \cup\{(1,1)\} \subseteq \mathbb{R}^{2}
$$

Glasgow Math. J. 21 (1980) 57-68. 
is a complete lattice, partially ordered componentwise. Here we have $\bigvee \mathfrak{B}(0,0)^{\downarrow}=(0,0)$ but $\wedge \mathfrak{B}(0,0)^{\uparrow}=(1,1)$.

Order convergence is said to be pre-topological on $L$ if $\mathfrak{B}(x) \longrightarrow x$ for all $x \in L$. The order topology of a lattice $L$ is defined to be the system $\mathfrak{O}=\mathscr{O}(L)$ of all subsets $Y$ of $L$ such that $x \in Y$ implies $Y \in \mathfrak{B}(x)$. It is easily verified that this is in fact the finest topology such that order convergence implies $\mathscr{D}$-convergence. In general, order convergence is distinct from $\mathfrak{D}$-convergence (see Example 1), and the neighbourhood filter $\mathfrak{U}(x)$ of $x$ in $\mathscr{D}$ may be properly contained in $\mathfrak{B}(x)$ (see Example 2 below; it is obvious that the inclusion $\mathfrak{U}(x) \subseteq \mathfrak{B}(x)$ is always fulfilled). Now order convergence is said to be topological if it agrees with convergence in the order topology. Clearly, this implies that order convergence is pre-topological, but the converse fails to be true as was pointed out by Kent in [10]:

EXAmpLE 2. The "right-open square"

$$
L=\left\{(x, y) \in[0,1]^{2}: 0<y<1 \text { implies } x<1\right\},
$$

together with componentwise order, is a complete lattice for which order convergence is pre-topological but not topological.

We bring together some trivial (and rather ineffective) characterizations of topological order convergence in

Proposition 1. For any lattice $L$, the following conditions are equivalent:

(a) order convergence is topological;

(b) there exists a topology $\mathfrak{I}$ such that order convergence coincides with $\mathfrak{T}$-convergence;

(c) for all $x \in L, \mathfrak{H}(x)$ order converges to $x$;

(d) order convergence is pre-topological, and $\mathfrak{U}(x)=\mathfrak{B}(x)$ for all $x \in L$.

A less trivial result is

Proposition 2. On a lattice $L$, order convergence is topological iff for all $x \in L, \mathfrak{H}(x)$ has a base of intervals.

This has been asserted by A. Gingras [7] for complete lattices, but his proof is based on the wrong hypothesis that the notions "pre-topological" and "topological" would coincide on complete lattices (cf. Example 2). A correct proof of Proposition 2 and other recent results on order convergence can be found in [5].

In [10], Kent has described pre-topological order convergence in terms of the ideals

$$
I(x)=\bigcap\{I: I \text { is an ideal with } \bigvee I=x\}
$$

and the dual ideals

$$
D(x)=\bigcap\{D: D \text { is a dual ideal with } \wedge D=x\}:
$$

PROPOSITION 3. The order convergence of a lattice $L$ is pre-topological iff

$$
x=\bigvee I(x)=\bigwedge D(x) \text { for all } x \in L \text {. }
$$


Whenever this is fulfilled, a base for the filter $\mathfrak{B}(x)$ is given by

$$
\{[y, z]: y \in I(x), z \in D(x)\} .
$$

A similar short description of topological order convergence seems to be unkown.

2. Characterization of order-topological lattices. A pair $(L, \mathfrak{I})$ is a topological lattice if $L$ is a lattice and $\mathfrak{T}$ a topology on $L$ such that the lattice operations

$$
\vee: L \times L \rightarrow L, \quad(x, y) \mapsto x \vee y
$$

and

$$
\wedge: L \times L \rightarrow L, \quad(x, y) \mapsto x \wedge y
$$

are continuous with respect to $\mathfrak{T}$. Thus $L$ is order-topological iff the order convergence on $L$ is topological and $(L, \mathfrak{O})$ is a topological lattice. An easy verification gives

Proposition 4. Every chain (complete or not) is an order-topological lattice.

Two other sufficient but not necessary conditions for a lattice to be order-topological have been established by Kent in [10]: Any lattice $L$ satisfying (c1) and

$$
x \in I(y) \text { or } y \in D(x) \text { implies } I(y) \cap D(x) \neq \varnothing
$$

or

$$
\begin{array}{lllll}
x \in I(y) & \text { and } & x \leq z & \text { implies } & x \in I(z), \\
x \in D(y) & \text { and } & z \leq x & \text { implies } & x \in D(z)
\end{array}
$$

is order-topological. However, neither (c2) nor (c3) are necessary for a lattice to be order-topological, as can be seen from Proposition 4 and the observation that there are chains for which both (c2) and (c3) are violated (whereas (c1) holds in every chain):

ExAmple 3 . In the complete chain $L=\{0\} \cup[1 / 2,1]$, linearly ordered by $\leq$, the element $x=1 / 2$ has the property $x \in I(x)=\{0,1 / 2\}$ while $I(x) \cap D(x)=\varnothing$, disproving (c2). For $1 / 2<y<1$, we have $y \in I(1)$ but not $y \in I(y)=\{0\} \cup[1 / 2, y[$, so (c3) fails as well.

Furthermore, a (complete) lattice with topological order convergence need not be order-topological, as becomes evident from a counterexample due to A. Gingras [7] (who also remarked that Kent's Example 2 in [10] is not correct):

Example 4. The set

$$
L=([0,1] \times\{1\}) \cup(\{0\} \times[0,1]) \cup\{(1,0)\},
$$

partially ordered by componentwise order, is a complete lattice with topological order convergence, but neither the join- nor the meet-operation is continuous at $x=(1,0)$ (see Proposition 5).

For any ideal $I$ of a lattice $L$ and all $x \in L, x \wedge I=x \downarrow \cap I$ is again an ideal. Now $L$ is called meet-continuous (written $\wedge$-continuous) if for every ideal $I$ in $L$ and all $x \in L$, $z=\bigvee I$ implies $x \wedge z=\bigvee(x \wedge I)$. Note that we do not postulate completeness for $L$. 
$\checkmark$-continuity is defined dually. The lattice in Example 4 is neither $\vee$ - nor $\wedge$-continuous.

In [4], it is shown that the preceding definition in fact characterizes continuity of certain intrinsic lattice operations:

Proposition 5. For a lattice $L$, the following four conditions are equivalent:

(a) $L$ is $\wedge$-continuous;

(b) the meet-operation $\wedge: L \times L \rightarrow L$ is continuous with respect to order convergence: $\mathfrak{\mho} \longrightarrow x$ and $\mathbb{S} \longrightarrow y$ implies $\mathfrak{\mho} \wedge(\mathfrak{S} \longrightarrow x \wedge y$ (where $\mathfrak{\lessgtr} \wedge(\mathfrak{S}$ is the filter generated by the sets $F \wedge G$ with $F \in \mathfrak{F}, G \in(\mathfrak{S})$;

(c) for all $x \in X$, the local meet-operation $\phi_{x}: L \rightarrow L, y \mapsto x \wedge y$ is continuous with respect to order convergence: $\mathfrak{\mho} \longrightarrow y$ implies $x \wedge \mathfrak{\mho} \longrightarrow x \wedge y$ (where $x \wedge \mathfrak{\mho}$ is the filter generated by the sets $x \wedge F$ with $F \in \mathfrak{F})$;

(d) the local meet-operations $\phi_{x}$ are continuous in the order topology.

Each of these statements holds whenever the meet-operation $\wedge: L \times L \rightarrow L$ is continuous with respect to the order topology.

Let us conclude our preparing considerations by introducing the sets

$$
\begin{aligned}
I^{*}(x) & =\{y \in L: x \in I(y)\} \\
D^{*}(x) & =\{y \in L: x \in D(y)\}
\end{aligned} \quad(x \in L) .
$$

We are now able to prove that the equivalence of pre-topological and topological order convergence, although being violated in certain lattices, holds for all $\vee$ - and $\wedge$-continuous lattices.

THEOREM 1. For a lattice $L$, the following three conditions are equivalent:

(a) $L$ is order-topological;

(b) $L$ is $\vee$ - and $\wedge$-continuous, and order convergence is pre-topological on $L$;

(c) $x=\bigvee I(x)=\wedge D(x)$ for all $x \in L$, and $x \leq y$ implies $I(x) \subseteq I(y), D(y) \subseteq D(x)$.

If one of these conditions holds then the sets $I^{*}(x)$ and $D^{*}(x)(x \in L)$ form a subbase for the order topology.

Proof. (a) $\Rightarrow$ (b) is clear by Proposition 5.

(b) $\Rightarrow$ (c). By Proposition 3, we have $x=\bigvee I(x)=\wedge D(x)$. Now suppose $x \leq y$. Then for any ideal $\mathrm{I}, y=\bigvee I$ implies

$$
x=x \wedge y=\bigvee(x \wedge I), \quad I(x) \subseteq x \wedge I \subseteq I .
$$

Thus $I(x) \subseteq I(y)$, and $D(y) \subseteq D(x)$ can be shown analogously.

(c) $\Rightarrow$ (a). First, we prove

(1) $I(x)=\bigcup\{I(z): z \in I(x)\}$ for all $x \in L$.

Clearly $z \in I(x)$ implies $z \leq x, I(z) \subseteq I(x)$. Also $\mathfrak{V}=\{I(z): z \in I(x)\}$ is a directed system of 
ideals since the coordination $x \mapsto I(x)$ is isotone and $I(x)$ is directed (being an ideal). Hence $I=\bigcup \mathfrak{V}$ is also an ideal, and $\bigvee I(z)=z$ for all $z$ implies

$$
\begin{aligned}
x & =\bigvee I(x)=\bigvee\{\bigvee I(z): z \in I(x)\} \\
& =\bigvee(\bigcup \mathfrak{Y})=\bigvee I .
\end{aligned}
$$

Thus $I(x) \subseteq I$, as desired.

Next, we show that for all $y, z \in L$,

(2) $I^{*}(y)$ and $D^{*}(z)$ are open in $\mathfrak{O}$.

Suppose $\mathfrak{F} \longrightarrow x \in I^{*}(y)$. Then $I=\mathfrak{F}^{\downarrow}$ is an ideal (cf. [5], [10]) with $\vee I=x$, and $y \in I(x)$ implies $y \in I(z)$ for some $z \in I(x)$, by (1). Now we obtain $z \in I^{*}(y)$ and $z \in I(x) \subseteq I=\Im_{5}^{\downarrow}$, so $z^{\uparrow} \subseteq I^{*}(y)$ and $z^{\uparrow} \in \mathfrak{\mho}$, whence $I^{*}(y) \in \mathfrak{F}$. For $D^{*}(z)$, we conclude analogously.

Third, we claim that

(3) $\mathfrak{S}(x)=\left\{I^{*}(y): y \in I(x)\right\} \cup\left\{D^{*}(z): z \in D(x)\right\}$ is a subbase for $\mathfrak{H}(x)=\mathfrak{B}(x)$.

By Proposition 3, order convergence is pre-topological, and

$$
\mathfrak{B}(x)=\{[y, z]: y \in I(x), z \in D(x)\}
$$

is a base for $\mathfrak{B}(x)$. But for $y \in I(x), z \in D(x)$, we have

$$
x \in I^{*}(y) \cap D^{*}(z) \subseteq y^{\uparrow} \cap z^{\downarrow}=[y, z],
$$

and by $(2), I^{*}(y) \cap D^{*}(z)$ is an open member of $\mathfrak{U}(x)$. Observing that the inclusion $\mathfrak{U}(x) \subseteq \mathfrak{B}(x)$ always holds, we see that $\mathfrak{U}(x)$ coincides with $\mathfrak{B}(x)$, and by Proposition 1 , order convergence is topological.

Finally, we prove

(4) $L$ is $\wedge$-continuous.

Let $I$ be an ideal possessing a join $y$. For any $x \in L, x \wedge y \leq y$ implies

$$
I(x \wedge y) \subseteq x^{\downarrow} \cap I(y) \subseteq x^{\downarrow} \cap I=x \wedge I .
$$

We have to verify that $x \wedge y$ is the least upper bound of the ideal $x \wedge I$. In fact, if $z$ is any upper bound of $x \wedge I$ then $x \wedge y=\bigvee I(x \wedge y) \leq z$ since $I(x \wedge y) \subseteq x \wedge I$. On the other hand, it is clear that $x \wedge y$ is itself an upper bound for $x \wedge I$. By dual arguments, we find that $L$ is also $\vee$-continuous.

Since order convergence is topological, the implication (a) $\Rightarrow$ (b) in Proposition 5 ensures that the meet operation is continuous with respect to the order topology, and by duality, the join operation is also continuous. Thus $(L, \mathfrak{O})$ is a topological lattice.

It is not hard to see that either of the conditions (c2) and (c3) implies

$$
\begin{aligned}
I(x \wedge y) & =I(x) \cap I(y), \\
D(x \vee y) & =D(x) \cap D(y)
\end{aligned}
$$

for all $x, y \in L$, so that Kent's results in [10] are immediate consequences of the 
implication (c) $\Rightarrow$ (a) in Theorem 1 :

Corollary 6. A lattice satisfying (c1) and (c2') is an order-topological lattice in which the order topology has a base of convex open sublattices.

In fact, $\left(\mathrm{c} 2^{\prime}\right)$ means that each $I^{*}(y)$ is a dual ideal and each $D^{*}(z)$ is an ideal whence the system

$$
\mathfrak{S}(x)=\left\{I^{*}(y) \cap D^{*}(z): y \in I(x), z \in D(x)\right\}
$$

is an open neighbourhood system consisting of convex sublattices. D. P. Strauss [16] has shown a powerful partial inversion of Corollary 6: if $(L, \mathfrak{T})$ is a compact $\left(\mathrm{T}_{2}\right)$ topological lattice with a base of open convex sublattices then $\mathfrak{I}$ must already coincide with the order topology (and with the interval topology). See also [5] and [13].

3. S-continuous lattices. In the last years, the so-called continuous lattices introduced by $\mathrm{D}$. Scott $[\mathbf{1 5}]$ have turned out to be a fundamental concept of modern lattice theory. Let us call a lattice S-continuous if

$$
x=\bigvee I_{S}(x) \text { for all } x \in L,
$$

where $I_{\mathrm{S}}(x)$ is the intersection of all ideals $I$ in $L$ possessing a join $z$ with $x \leq z$. The complete S-continuous lattices are exactly the continuous lattices in the sense of Scott. An easy computation shows

Proposition 7. In a $\wedge$-continuous lattice $L$, the ideal $I(x)$ coincides with $I_{\mathrm{S}}(x)$, for each $x \in L$.

COROLlaRy 8 . For a lattice $L$, the following three conditions are equivalent:

(a) $L$ is S-continuous;

(b) $L$ is $\wedge$-continuous, and $x=\bigvee I(x)$ for all $x \in L$;

(c) $x=\bigvee I(x)$ for all $x \in L$, and $x \leq y$ implies $I(x) \subseteq I(y)$.

Proof. (a) $\Rightarrow$ (c). The inclusion $I_{\mathrm{S}}(x) \subseteq I(x) \subseteq x^{\downarrow}$ always holds, so $x=\bigvee I_{\mathrm{S}}(x)$ implies $I(x)=I_{\mathrm{S}}(x)$ and $x=\bigvee I(x)$. If $x \leq y$ and $I$ is any ideal with $y=\bigvee I$ then $I(x)=I_{\mathrm{S}}(x) \subseteq I$. Hence $I(x)$ is contained in $I(y)$.

(c) $\Rightarrow$ (b). See Part (4) in the proof of Theorem 1.

(b) $\Rightarrow$ (a). Proposition 7.

Now from Theorem 1 , we derive immediately

Theorem 2. A lattice $L$ is order-topological iff $L$ and the dual lattice $L^{*}$ are S-continuous.

By an upper segment of $L$, we mean a subset $U$ with $x^{\uparrow} \subseteq U$ for all $x \in L$. The $\subseteq(L)$-open upper segments form a topology $\sigma(L)$, the so-called Scott-topology (Stopology). In [4] and [5], it is shown that a set $U$ is $\mathfrak{O}(L)$-open iff for every ideal $I$ and for every dual ideal $D$ with $\bigvee I=\wedge D \in U$, there exist $y \in I$ and $z \in D$ such that the whole 
interval $[y, z]=y^{\uparrow} \cap z^{\downarrow}$ is contained in $U$. From this observation, one easily derives that an upper segment $U$ is $\mathfrak{O}(L)$-open (hence $\sigma(L)$-open) iff it intersects every ideal which has a join contained in $U$. The bi-S-topology $\mathscr{O}_{\mathbb{S}}(L)$ is the coarsest topology comprehending $\sigma(L)$ and the S-topology $\sigma\left(L^{*}\right)$ of the dual lattice $L^{*}$. Thus $\mathfrak{O}_{\mathrm{S}}(L)$ has a base of convex sets (moreover, a subbase of upper and lower segments) and is always contained in the order topology $\mathscr{O}(L)$. However, $\mathfrak{O}_{\mathrm{S}}(L)$ may be even strictly contained in the so-called convex order topology $\mathfrak{O}_{C}(L)$ which has as a base the set of all convex $\subseteq(L)$-open sets (cf. [13]).

EXAMPLe 5. Let $I$ denote the real unit interval and $K$ the complete Boolean lattice of all regular open subsets of $I$. Form the disjoint union of $I$ and $K$, and identify the corresponding greatest and least elements, respectively. Thereby, a complete lattice $L$ is obtained in which the set $Y=I \backslash\{0,1\}$ is convex and open in $\mathfrak{D}_{C}(L)$ but not in $\mathfrak{D}_{S}(L)$ since every nonempty $\sigma(L)$-open set intersects every nonempty $\sigma\left(L^{*}\right)$-open set outside from $Y$ (cf. E. E. Floyd [6]).

This example also shows that a filter may converge to $x$ both in $\sigma(L)$ and in $\sigma\left(L^{*}\right)$ but not in the order topology $\subseteq(L)$ (otherwise, $\subseteq(L)$ would always agree with $\mathfrak{D}_{\mathrm{S}}(L)$ ).

In an order-topological lattice, each of the sets $I^{*}(y)$ is an $\subseteq(L)$-open upper segment, i.e. a $\sigma(L)$-open set. Dually, each $D^{*}(z)$ is $\sigma\left(L^{*}\right)$-open. In particular, the order topology has a base of $\mathfrak{D}_{\mathrm{S}}(L)$-open sets, and we infer

Corollary 9. In an order-topological lattice $L$, the bi-S-topology $\mathfrak{O}_{\mathrm{S}}(L)$ coincides with the order topology $\mathfrak{D}(L)$, and, consequently, the following three conditions are equivalent for a filter $\widetilde{\wp}$ on $L$ :

(a) $\mathfrak{z}$ order converges to $x$ in $L$;

(b) $\mathfrak{乛}$ converges to $x$ in $\subseteq(L)$;

(c) $\widetilde{\mho}$ converges to $x$ in $\sigma(L)$ and in $\sigma\left(L^{*}\right)$.

Similarly, one can prove that in a $\vee$ - and $\wedge$-continuous lattice $L$, the topologies $\mathfrak{D}_{\mathrm{S}}(L)$ and $\mathfrak{Q}_{\mathrm{C}}(L)$ coincide.

Observing that every completely distributive lattice is S-continuous (see [16], p. 38) and that complete distributivity is a selfdual property, we derive from Theorem 2.

CoRollary 10. Every completely distributive lattice is order-topological. Moreover, it is a compact $\mathrm{T}_{2}$ space in its order topology which agrees with the interval topology.

In fact, every ultrafilter on a completely distributive lattice has a unique order limit [17], and since order convergence is topological, the order topology must be compact and $\mathrm{T}_{2}$. The last statement is due to A. J. Ward [18].

4. Compactly generated lattices. An element $x$ of a lattice $L$ (not necessarily complete) is called compact if it is contained in every ideal $I$ possessing a join $y$ with $x \leq y$. The dual notion is cocompact. A lattice $L$ is compactly generated if every element of $L$ is a join of compact elements. (Again, we do not postulate completeness for $L$, contrary to the convention in [3]). If both $L$ and $L^{*}$ are compactly generated then we speak of a bicompactly generated lattice. We collect some obvious characterizations of compact 
elements in

Proposition 11. The following five conditions are equivalent:

(a) $x$ is compact;

(b) $x \in I_{\mathrm{s}}(x)$;

(c) $I^{*}(x)=x^{\uparrow}$;

(d) $x^{\uparrow}$ is not only closed but also open in the order topology;

(e) $x^{\uparrow}$ is open in the Scott-topology.

Notice that every principal ideal $x^{\downarrow}$ and every principal dual ideal $x^{\uparrow}$ is not only closed in the order topology but also in the coarser interval topology. On the contrary, $x^{\uparrow}$ is not closed in the Scott-topology unless $x$ is the only element of $L$, while $x^{\uparrow}$ is always Scott-closed (being the complement of an upper segment).

A straightforward calculation shows

Proposition 12. (1) A lattice $L$ is compactly generated iff for all $x, y \in L$ with $x<y$, there exists a compact element $u$ such that $u \leq y$ but not $u \leq x$.

(2) In a compactly generated lattice, the ideal $I(x)$ is generated by the compact elements below $x$. Furthermore, $x=\bigvee I(x)$, and $I(x \wedge y)=I(x) \cap I(y)$.

(3) Every compactly generated lattice is $S$-continuous.

Moreover, one can show

THEOREM 3. For a lattice $L$, the following four conditions are equivalent:

(a) $L$ is bicompactly generated;

(b) The system $\mathfrak{A}=\left\{y^{\uparrow}: y\right.$ is compact $\} \cup\left\{z^{\downarrow}: z\right.$ is cocompact $\}$ is a subbase for the order topology of $L$;

(c) The order topology of $L$ has a subbase consisting of principal ideals and principal dual ideals;

(d) $L$ is $\vee$ - and $\wedge$-continuous, and the order topology of $L$ has an open base consisting of closed intervals.

If one of these conditions holds then $L$ is order-topological and satisfies (c1), (c2') and (c3). Moreover, $L$ is a zero-dimensional regular space in its order topology (possessing a base of closed-open sets).

Proof. (a) $\Rightarrow$ (b) by Theorem 1, Proposition 11 and Proposition 12.

(b) $\Rightarrow$ (c). Trivial.

(c) $\Rightarrow$ (a). For $x<y$, there exist $u^{\uparrow}, v^{\downarrow} \in D_{\text {with }} y \in[u, v]=u^{\uparrow} \cap v^{\downarrow}, x \notin[u, v]$ (since the order topology is always $\mathrm{T}_{1}$ ). It follows that $u \leq y, x \leq v, u \neq x$. But, by Proposition 11, $u$ is compact, and applying Proposition 12.(1), we conclude that $L$ is compactly generated, and by duality, so is $L^{*}$.

(a) $\Rightarrow$ (d). Clear by Proposition 12 Part (3) and the implication (a) $\Rightarrow$ (c).

(d) $\Rightarrow$ (c). It suffices to show that for any $\mathcal{Q}$-open interval $[u, v]=u^{\uparrow} \cap v^{\downarrow}, u^{\uparrow}$ and $v^{\downarrow}$ are also open. By Proposition 5, the mapping $\phi_{v}: y \mapsto y \wedge v$ is continuous, and, consequently, $u^{\uparrow}=\phi_{v}^{-1}([u, v])$ is open in $\mathfrak{O}$. By duality, the same holds for $v^{\downarrow}$. 
The other statements are clear (cf. [1], [11]). As a trivial consequence of Theorem 3, we notice

COROLLARY 14. Every lattice without infinite chains is order-topological, and its order topology is discrete.

5. Boolean lattices. By a Brouwerian lattice (or a Heyting algebra) there is meant a lattice $L$ with least element 0 such that each element $x$ of $L$ has a pseudo-complement $x^{\prime}$ satisfying the equivalence

$$
y \leq x^{\prime} \Leftrightarrow x \wedge y=0 .
$$

(In other words, the sets $\{y \in L: x \wedge y=0\}$ are principal ideals). The following result is well-known for complete lattices:

Proposition 15. Every Brouwerian lattice is $\wedge$-continuous. In particular, every Boolean lattice is $\vee$ - and $\wedge$-continuous.

One can show somewhat more: if $L$ is a Brouwerian lattice and $Y$ is an arbitrary subset of $L$ with join $z$ then for all $x \in L, x \wedge z$ is the join of the set $x \wedge Y$. (See [2, p. 174].)

In Boolean lattices, it is possible to describe the ideals $I(x)$ explicitly in terms of atoms:

Proposition 16. In a Boolean lattice $L$, the ideal $I(x)$ consists of all finite joins of atoms below $x$. In particular, $I(1)$ is the ideal generated by the atoms of $L$. (If there is no atom below $x$ then $I(x)=\{\bigvee \varnothing\}=\{0\}$.)

Proof. In order to show that $I(x)$ is generated by the atoms below $x$, we may assume that $x=1$ (since $I(x)$ is also the intersection of all ideals in the Boolean sublattice $[0, x]$ with join $x$ ). Let $I$ be any ideal with $\vee I=1$. If there exists an atom $a$ with $a \notin I$ then $I \subseteq a^{\prime \downarrow}$, and it follows that $1=\bigvee I \leq a^{\prime}$, a contradiction. Hence $I(1)$ contains all atoms. Conversely, assume $y \in I(1)$, and let $I$ denote the ideal generated by the atoms of $L$. Furthermore, let $P$ be an arbitrary prime ideal containing $I$. If 1 is not the join of $P$ then there exists an upper bound $z$ of $P$ with $z<1$. Thus $P \subseteq z^{\downarrow}$, and by the maximality of $P$, equality holds, and $z$ is a dual atom. But then $z^{\prime}$ is an atom belonging to $P$, which is impossible because $z^{\prime}$ cannot be dominated by $z$. Accordingly, we have $\vee P=1, y \in P$, and it follows that $I(1)$ is a subset of the intersection of all prime ideals containing $I$. But this intersection coincides with $I$, on account of the Prime Ideal Theorem. Finally, it is clear that $I$ consists of all finite joins formed by atoms of $L$.

Corollary 17. Every Boolean lattice satisfies condition (c3), and the ideal $I=I(1)$ consists of all compact elements. Furthermore, $I$ is the intersection of all non-principal ideals.

Proof. In a Boolean lattice, the compact elements are precisely the finite joins of atoms. By relativization, we find that $I(x)$ consists of all compact elements below $x$, and this implies the first half of (c3). The second follows analogously, by observing that $L$ is 
selfdual. If $P$ is a non-principal prime ideal then $\bigvee P=1$, and $I$ is contained in $P$. On the other hand, as we have seen before, $I$ cannot be contained in any principal prime ideal (which would be generated by a dual atom).

As a little application, we obtain the well-known fact that the filter of all co-finite subsets of an infinite set is the intersection of all non-principal ultrafilters (cf. [14]).

Another immediate consequence of Proposition 16 is

Corollary 18. A Boolean lattice $L$ has no atoms iff $I(x)=\{0\}$ for all $x \in L$. Hence for no (non-trivial) atomless Boolean lattice can order convergence be pre-topological.

Examples of such atomless (complete) Boolean lattices are the lattices of all regular open subsets of $\mathbb{R}$ and of all measurable sets modulo null sets, respectively (cf. [18]).

Now we are in position to give a lot of alternative characterizations of ordertopological Boolean lattices:

THEOREM 4. For a Boolean lattice $L$, the following conditions are all equivalent:

(a) $\mathrm{L}$ is S-continuous;

(b) $L$ is atomic (i.e. every non-zero element dominates at least one atom);

(c) $L$ is a point lattice (i.e. every element is a join of atoms);

(d) 1 is a join of atoms;

(e) $L$ is compactly generated;

(f) $x=\bigvee I(x)$ for all $x \in L$;

(g) Order convergence is pre-topological on $L$;

(h) Order convergence is topological on $L$;

(i) $\left\{a^{\uparrow}: a\right.$ is an atom of $\left.L\right\} \cup\left\{z^{\downarrow}: z\right.$ is a dual atom of $\left.L\right\}$ is a subbase for the order topology;

(j) $\left\{a^{\dagger}: a\right.$ is an atom of $\left.L\right\}$ is a subbase for the Scott-topology;

(k) $L$ is order-topological.

Proof. The equivalence of the first five purely order-theoretical statements is wellknown (at least in the case of complete lattices, cf. [3, pp. 32-35] and [16, p. 59]).

(e) $\Leftrightarrow$ (f). Proposition 16 and Corollary 17 .

(f) $\Leftrightarrow$ (g). Propositions 3. (Recall that a Boolean lattice is selfdual).

$(\mathrm{g}) \Leftrightarrow(\mathrm{h}) \Leftrightarrow(\mathrm{k})$. Theorem 1 and Proposition 15 .

(e) $\Rightarrow$ (i). By Theorem 3, the set

$$
\mathfrak{H}=\left\{y^{\uparrow}: y \text { is compact in } L\right\} \cup\left\{z^{\downarrow}: z \text { is cocompact in } L\right\}
$$

is a subbase for the order topology. But any compact element $y$ is a finite join of atoms, say $y=\bigvee A_{y}$. Thus $y^{\uparrow}=\bigcap\left\{a^{\uparrow}: a \in A_{y}\right\}$, and a dual argument holds for dual atoms.

(i) $\Rightarrow$ (e). Theorem 3 .

$(\mathrm{e}) \Leftrightarrow(\mathrm{j})$ Analogously.

COROLlaRy 19. For a complete Boolean lattice $L$, the following conditions are 
equivalent:

(a) $L$ is atomic;

(b) $L$ is completely distributive;

(c) $L$ is isomorphic to a power set;

(d) $L$ is continuous in the sense of Scott;

(e) $L$ is order-topological;

(f) $L$ is a Boolean space in its interval topology (which coincides with the order topology);

(g) $L$ is a $T_{2}$ space in its interval topology.

Proof. The equivalence of (a); (b) and (c) is a standard result of lattice theory (cf. [3, p. 35]). As M. Katětov has shown in [8], a Boolean lattice is atomic iff its interval topology is $T_{2}$. An application of Corollary 10, Theorem 3 and Theorem 4 completes the proof. (Recall that a Boolean space is a totally disconnected compact $T_{2}$ space.)

It should be emphasized that an atomic Boolean lattice need not be complete. (For example, the lattice of all finite and cofinite subsets of an infinite set is not). The order topology of an atomic Boolean lattice is compact if and only if the lattice is complete. In [11], this additional requirement has not been stated explicitly. In the same paper, it has been shown that the order topology of a complete atomic Boolean lattice is uniformizable. But this is now clear since a compact $T_{2}$ space is always uniformizable.

6. Concluding remarks and problems. We have seen in Proposition 5 that every lattice which is a topological lattice in its order topology must be $\vee$ - and $\wedge$-continuous. We wonder if the converse is also true. Probably, a counterexample is the complete atomless Boolean lattice of all regular open subsets of $\mathbb{R}$ (and it may be that every atomless Boolean lattice is a counterexample). However, it can be shown that a $\vee$ - and $\wedge$ continuous lattice $L$ for which the order topology $\cong(L \times L)$ coincides with the product topology $\mathscr{O}(L) \otimes \mathfrak{O}(L)$ is a topological lattice with respect to the order topology. It seems to be a deeper problem to classify those lattices $L$ for which the identity

$$
\Im(L \times L)=\bigcirc(L) \otimes Ð(L)
$$

is valid (cf. [4]).

The equivalence of pre-topological and topological order convergence (resp. Sconvergence) in $\vee$ - and $\wedge$-continuous lattices has an analogue in the theory of topological groups: if $G$ is a group with a pre-topological convergence function for which the group operations are continuous then $G$ is already a topological group (cf. H.-J. Kowalsky [12]). However, it should be emphasized that we have derived the corresponding result for lattices only in one special case, namely that of order convergence. It is conjectured that an arbitrary pre-topological lattice need not be topological.

Finally, it should be mentioned that the generalization of notions like compactness and continuity from complete to arbitrary lattices proposed in this paper is not the only one which allows us to extend many "classical" results on complete lattices. Another "natural" possibility (which even works in arbitrary posets) is to replace all statements of 
the form $x \leq \bigvee Y$ by $x \in Y^{\uparrow \downarrow}$ and all identities $x=\bigvee Y$ by $x^{\downarrow}=Y^{\uparrow \downarrow}$. This alternative procedure will be discussed elsewhere.

\section{REFERENCES}

1. C. R. Atherton, Concerning intrinsic topologies on Boolean algebras and certain bicompactly generated lattices, Glasgow Math. J. 11 (1970), 156-161.

2. R. Balbes and Ph. Dwinger, Distributive lattices (University of Missouri Press, Columbia, Missouri, 1974).

3. P. Crawley and R. P. Dilworth, Algebraic theory of lattices (Prentice Hall, 1973).

4. M. Erné, Topologies on products of partially ordered sets III: Order convergence and order topology, to appear in Algebra Universalis.

5. M. Erné and S. Weck, Order convergence in lattices, to appear in Rocky Mountain J. Math. $687-689$

6. E. E. Floyd, Boolean algebras with pathological order topologies, Pacific J. Math. 5 (1955),

7. A. Gingras, Order convergence and order ideals, Proceedings of the special session on convergence structures (Univ. of Nevada, Reno, 1976), 45-49.

8. M. Katětov, Remarks on Boolean algebras, Colloq. Math. 2 (1951), 229-235.

9. D. C. Kent, Convergence functions and their related topologies, Fund. Math. 54 (1964), 125-133.

10. D. C. Kent, On the order topology in a lattice, Illinois J. Math. 10 (1966), 90-96.

11. D. C. Kent and C. R. Atherton, The order topology in a bicompactly generated lattice, J.

12. H.-J. Kowalsky, Beiträge zur topologischen Algebra, Math. Nachr. 11 (1954), 143-185.

13. J. D. Lawson, Intrinsic topologies in topological lattices and semilattices, Pacific J. Math. Austral. Math. Soc. 8 (1968), 345-349.

32 (1970), 459-466.

14. J. Schmidt, Beiträge zur Filtertheorie I, Math. Nachr. 7 (1952), 359-378.

15. D. Scott, Continuous lattices, Toposes, algebraic geometry and logic, Lecture Notes in Mathematics No. 274 (Springer-Verlag, 1972).

16. Seminar on continuity in (semi-)lattices: A compendium of continuous lattices (TH Darmstadt 1978).

17. D. P. Strauss, Topological lattices, Proc. London Math. Soc. 18 (1968), 217-230.

18. A. J. Ward, On relations between certain intrinsic topologies in partially ordered sets, Proc. Cambridge Philos. Soc. 51 (1955), 254-261.

INSTITUT FÜr MATHEMATIK

UNIVERSITÄT HANNOVER

D 3 HANNOVER

Fed. Rep. of Germany 